DOI: $10.18027 / 2224-5057-2021-11-3 s 2-10$

Цитирование: Тюляндина А.С., Коломиец Л.А., Морхов К.Ю., Нечушкина В.М., Покатаев И.А., Румянцев А.А. и соавт. Практические рекомендации по лекарственному лечению рака яичников, первичного рака брюшины и рака маточных труб. Злокачественные опухоли: Практические рекомендации RUSSCO \#3s2, 2021 (том 11). 10

\title{
ПРАКТИЧЕСКИЕ РЕКОМЕНДАЦИИ ПО ЛЕКАРСТВЕННОМУ ЛЕЧЕНИЮ РАКА ЯИЧНИКОВ, ПЕРВИЧНОГО РАКА БРЮШИНЫ И РАКА МАТОЧНЫХ ТРУБ
}

Коллектив авторов: Тюляндина А.С., Коломиец Л.А., Морхов К.Ю., Нечушкина В.М., Покатаев И.А., Румянцев А.А., Тюляндин С.А., Урманчеева А.Ф., Хохлова С.В.

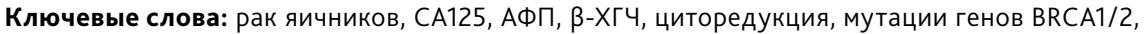
химиотерапия, рецидив

Клиническое течение и методы лечения первичного рака брюшины и рака маточных труб аналогичны таковым при раке яичников (РЯ), в связи с этим все три нозологические формы рассматриваются совместно в одном разделе.

\section{1. ОПРЕДЕЛЕНИЕ СТАДИИ}

Таблица 1. Система стадирования рака яичников по TNM и FIGO (8-е издание, 2016 г.)

\begin{tabular}{|c|c|c|}
\hline TNM & FIGO & \\
\hline T1 & I & Опухоль ограничена яичниками \\
\hline T1a & $\mathrm{IA}$ & $\begin{array}{l}\text { Опухоль ограничена одним яичником, капсула не повреждена, нет опухолевых } \\
\text { разрастаний на поверхности яичника, нет злокачественных клеток в асцитической } \\
\text { жидкости или смывах из брюшной полости }\end{array}$ \\
\hline T1b & IB & $\begin{array}{l}\text { Опухоль ограничена двумя яичниками, их капсулы не повреждены, нет опухолевых } \\
\text { разрастаний на поверхности яичников, нет злокачественных клеток в асцитической } \\
\text { жидкости или смывах из брюшной полости }\end{array}$ \\
\hline T1c & IC & $\begin{array}{l}\text { Опухоль ограничена одним или двумя яичниками и сопровождается любым из } \\
\text { следующих факторов }\end{array}$ \\
\hline T1c1 & IC1 & Повреждение капсулы во время операции \\
\hline T1c2 & IC2 & $\begin{array}{l}\text { Повреждение капсулы до операции или опухоль на поверхности яичника/маточной } \\
\text { трубы }\end{array}$ \\
\hline T1c3 & IC3 & Злокачественные клетки в асцитической жидкости или смывах с брюшины \\
\hline $\mathrm{T} 2$ & II & Опухоль поражает один или два яичника с распространением на малый таз \\
\hline $\mathrm{T} 2 \mathrm{a}$ & $\| \mathrm{A}$ & Врастание и/или метастазирование в матку и/или в одну или обе маточные трубы \\
\hline
\end{tabular}




\begin{tabular}{|c|c|c|}
\hline TNM & FIGO & \\
\hline $\mathrm{T} 2 \mathrm{~b}$ & IIB & Распространение на другие ткани таза \\
\hline $\begin{array}{l}\text { Т3 и/или } \\
\text { N1 }\end{array}$ & III & $\begin{array}{l}\text { Опухоль поражает один или оба яичника с гистологически подтвержденными } \\
\text { внутрибрюшинными метастазами за пределами таза и/или метастазами в регионар- } \\
\text { ных лимфатических узлах (внутренних, наружных и общих подвздошных, запиратель- } \\
\text { ных, крестцовых или поясничных лимфоузлах) }\end{array}$ \\
\hline \multirow[t]{3}{*}{ N1 } & IIIA1 & Метастазы только в забрюшинных лимфатических узлах \\
\hline & IIIA1 (i) & Метастазы в лимфатических узлах размерами до 10 мм \\
\hline & IIIA (ii) & Метастазы в лимфатических узлах размерами более 10 мм \\
\hline T3a & IIIA2 & $\begin{array}{l}\text { Микроскопические гистологически подтвержденные внутрибрюшинные метастазы } \\
\text { за пределами таза } \pm \text { метастазы в забрюшинных лимфатических узлах }\end{array}$ \\
\hline $\mathrm{T} 3 \mathrm{~b}$ & IIIB & $\begin{array}{l}\text { Макроскопические внутрибрюшинные метастазы за пределами таза до } 2 \text { см включи- } \\
\text { тельно в наибольшем измерении } \pm \text { метастазы в забрюшинных лимфатических узлах }\end{array}$ \\
\hline $\mathrm{T} 3 \mathrm{C}$ & IIIC & $\begin{array}{l}\text { Внутрибрюшинные метастазы за пределами таза более } 2 \text { см в наибольшем измерении } \\
\pm \text { метастазы в забрюшинных лимфатических узлах (включая распространение } \\
\text { опухоли на капсулу печени и селезенки без поражения паренхимы органов) }\end{array}$ \\
\hline \multirow[t]{3}{*}{ M1 } & IV & Отдаленные метастазы (исключая внутрибрюшинные метастазы) \\
\hline & IVA & Плевральный выпот со злокачественными клетками \\
\hline & IVB & $\begin{array}{l}\text { Метастазы в паренхиматозных органах и других органах вне брюшной полости (в том } \\
\text { числе паховых лимфатических узлах и лимфатических узлах за пределами брюшной } \\
\text { полости) }\end{array}$ \\
\hline
\end{tabular}

\section{2. ДИАГНОСТИКА}

Диагноз РЯ устанавливается на основании гистологического исследования материала, полученного во время операции или при биопсии на дооперационном этапе. При невозможности выполнения на первом этапе оперативного вмешательства или биопсии лечение начинается с предоперационной ХT, в этом случае диагноз может быть установлен на основании данных цитологического исследования, уровня СА125 и клинической картины. К злокачественным эпителиальным опухолям яичников относят следующие гистологические типы (классификация ВОЗ, 4-е издание, 2014 г.):

- серозная карцинома;

- низкой степени злокачественности (low grade);

- высокой степени злокачественности (high grade);

- эндометриоидная карцинома;

- муцинозная карцинома;

- светлоклеточная карцинома;

- злокачественная опухоль Бреннера;

- серозно-муцинозная карцинома;

- недифференцированная карцинома;

- смешанная эпителиальная карцинома. 
Классификацией ВОЗ предусматривается разделение серозного гистологического подтипа на две степени злокачественности - низкую (low grade) и высокую (high grade), в то время как для оценки эндометриоидных и муцинозных опухолей следует использовать трехуровневую систему оценки степени злокачественности (опухоли низкой, умеренной и высокой степени злокачественности). Степень злокачественности опухоли обязательно должна быть отражена в патоморфологическом заключении, так как это может иметь значение для определения тактики лечения".

Объем обследования:

- осмотр, сбор анамнеза болезни, семейного анамнеза;

- клинический анализ крови;

- биохимический анализ крови с показателями функции печени, почек;

- коагулограмма;

- определение концентрации СА125 в плазме крови;

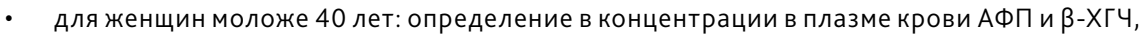
лДГ для исключения герминогенных опухолей, а также ингибина - для исключения стромальных опухолей;

- УзИ органов брюшной полости и малого таза, шейно-надключичных, паховых областей и забрюшинного пространства, вен нижних конечностей по показаниям;

- рентгенография органов грудной клетки;

- ЭГДС;

- колоноскопия;

- КТ органов грудной клетки, брюшной полости и малого таза с в/в контрастированием и/или МРТ брюшной полости и малого таза св/в контрастированием с целью оценки возможности оптимальной циторедуктивной операции и/или ПЭТ-КТ (данный метод не следует использовать для оценки эффективности ХT и в процессе наблюдения, рекомендуется только для оценки степени распространенности опухолевого процесса при решения и вопроса о его резектабельности);

- для женщин старше 40 лет: маммография для исключения рака молочной железы с метастазами в яичниках;

- определение концентрации HE-4 в плазме крови и определение индекса ROMA при отсутствии морфологической верификации диагноза;

- определение РЭА и СА19-9 в крови при подозрении на муцинозную карциному.

При многоцикловом лечении необходимые показатели для мониторинга определяются перед каждым курсом XT, перечень и сроки их выполнения подробно описаны в главе по общим принципам проведения противоопухолевой терапии (пункт 2.6).

Всем больным с серозными и эндометриоидными карциномами высокой степени злокачественности показано определение патогенных мутации генов BRCA1/2 в образце крови и/или опухоли методом ПЦР для поиска типичных наследственных мутаций. При

1 Режим/nрепарат зарегистрирован в РФ, но не входит в клинические рекомендации, одобренные Минздравом РФ. 
отрицательном результате данного метода необходимо выполнение полного геномного секвенирования опухолевого образца для выявления редких герминальных и соматических мутаций в генах BRCA1/2. У больных с герминальными мутациями желательно проведение генетического консультирования.

Возможно определение в опухолевом образце MSI методом ИГХ для планирования иммунотерапии'.

Окончательное стадирование по TNM и FIGO осуществляется после лапаротомии (табл. 1).

\section{3. ЛЕЧЕНИЕ ПЕРВИЧНОГО РАКА ЯИЧНИКОВ, РАКА БРЮШИНЫ И РАКА МАТОЧНЫХ ТРУБ}

\section{1. Хирургическое лечение и хирургическое стадирование}

Лечение всех стадий РЯ целесообразно начинать с выполнения полной или оптимальной циторедуктивной операции. Полная циторедукция включает экстирпациюматки с придатками, удаление большого сальника на уровне большой кривизны желудка и всех видимых проявлений опухолевого процесса и означает отсутствие макроскопически определяемой остаточной опухоли. Оптимальная циторедукция включает экстирпацию матки с придатками, удаление большого сальника и видимых проявлений опухолевого процесса и допускает наличие остаточных опухолевых узлов, размеры каждого из которых $\leq 10$ мм. Неоптимальная циторедукция включает экстирпацию матки с придатками, удаление большого сальника, а также видимых проявлений опухолевого процесса, при этом размеры хотя бы одного из макроскопически определяемых остаточных опухолевых очагов >10 мм в диаметре.

Выполнение лимфаденэктомии у больных III-IV стадиями целесообразно при полной или оптимальной циторедукции в случае подозрения на метастатическое поражение лимфоузлов по данным предоперационного обследования или интраоперационной ревизии. Удалению подлежат внутренние, наружные, общие подвздошные, запирательные, преаортальные, парааортальные, аортокавальные, прекавальные и паракавальные, крестцовые лимфоузлы до уровня почечных сосудов.

Протокол операции должен содержать информацию об остаточной опухоли (размеры, количество очагов, локализация).

При визуальной картине I-ІІ стадий обязательно выполнение процедур хирургического стадирования, которое включает следующее:

- выполняют срединную лапаротомию с обходом пупка слева для полноценной ревизии органов брюшной полости и малого таза и адекватного доступа к большому сальнику;

- при отсутствии морфологической верификации диагноза во время операции следует проводить срочное морфологическое исследование;

1 Режим/nрепарат зарегистрирован в РФ, но не входит в клинические рекомендации, одобренные Минздравом РФ. 
- при обнаружении асцита жидкость аспирируют для цитологического исследования; при отсутствии асцита выполняют цитологическое исследование смывов с брюшины, в том числе с куполов диафрагмы, латеральных каналов и малого таза;

- проводят тщательную ревизию всех отделов брюшной полости, в том числе поддиафрагмального пространства, большого и малого сальника, тонкой и толстой кишок и их брыжеек, париетальной и висцеральной брюшины, а также забрюшинного пространства, все подозрительные участки подвергают биопсии; должна быть осмотрена вся поверхность брюшины; спаечный процесс, препятствующий ревизии, должен быть отмечен;

- в отсутствие диссеминации по данным ревизии выполняют биопсию брюшины прямокишечно-маточного углубления, мочевого пузыря, стенок малого таза, латеральных каналов, а также правого и левого куполов диафрагмы;

- выполняют тотальную тазовую и поясничную лимфаденэктомию до уровня почечных сосудов;

- удаляют большой сальник на уровне большой кривизны желудка;

- удаление аппендикса целесообразно при муцинозном раке и при подозрении на его поражение.

Органосохраняющее хирургическое вмешательство (односторонняя аднексэктомия с резекцией второго яичника, оментэктомия, тазовая и поясничная лимфаденэктомия и выполнение процедур стадирования) в виде исключения возможно у молодых больных, желающих сохранить фертильность, при высокодифференцированных серозной, эндометриоидной или муцинозной карциномах IA и IC1 стадий при наличии возможности тщательного диспансерного наблюдения и доказанном отсутствии наследственного характера заболевания. При эндометриоидном гистологическом варианте в этих случаях следует выполнить биопсию эндометрия для исключения синхронного рака эндометрия.

При невыполнении процедур стадирования при I-ІІ стадии во время первой операции рекомендуется выполнение повторной операции с целью стадирования.

Малоинвазивные хирургические вмешательства, в частности лапароскопические, могут быть использованы только для верификации диагноза, определения стадии или для прогнозирования объема циторедуктивной операции.

Операция «second-look» не рекомендуется.

\section{2. Лекарственное лечение}

\subsection{1. ААъювантная и лечебная химиотерапия}

Решение вопроса о проведении ХТ после операции основано на информации о стадии процесса, морфологическом варианте опухоли и степени злокачественности (табл. 2). 
Таблица 2. Алгоритм назначения химиотерапии в зависимости от стадии и морфологических особенностей опухоли

\begin{tabular}{|c|c|}
\hline Стадия и морфологические особенности опухоли & Вариант лечения \\
\hline $\begin{array}{l}\text { IA и IB стадии в сочетании с несветлоклеточным } \\
\text { гистологическим типом и низкой степенью } \\
\text { злокачественности }\end{array}$ & Адъювантная XТ не показана \\
\hline $\begin{array}{l}\text { IA и IB стадии в сочетании со светлоклеточным } \\
\text { гистологическим типом или высокой степенью } \\
\text { злокачественности }\end{array}$ & 4-6 циклов адъювантной платиносодержащей XТ \\
\hline $\begin{array}{l}\text { IA и IB стадии при отсутствии процедур полного } \\
\text { хирургического стадирования }\end{array}$ & $\begin{array}{l}6 \text { циклов адъювантной платиносодержащей ХТ } \\
\text { или повторное оперативное вмешательство с пол- } \\
\text { ным хирургическим стадированием с последующей } \\
\text { XТ по показаниям }\end{array}$ \\
\hline $\begin{array}{l}\text { IC-IV стадии вне зависимости от гистологического } \\
\text { типа опухоли }\end{array}$ & 6 циклов лечебной платиносодержащей XТ \\
\hline
\end{tabular}

При невозможности выполнения на первом этапе хирургического вмешательства в оптимальном объеме (неудовлетворительное общее состояние вследствие значительного распространения опухолевого процесса, серьезная сопутствующая патология) следует начинать лечение с XT с последующей промежуточной циторедуктивной операцией в максимально короткие сроки (после 2-3 циклов ХТ). Количество курсов предоперационной ХТ не должно быть более 4-х. После операции проводятся дополнительно 3-4 цикла ХТ по той же схеме вне зависимости от выраженности лечебного патоморфоза. В случае прогрессирования в процессе предоперационной ХТ возможен отказ от выполнения интервальной циторедукции.

Использование внутрибрюшинной гипертермической химиоперфузии (HIPEC) не рекомендуется в рутинной клинической практике.

В табл. 3 представлены рекомендуемые режимы адъювантной и лечебной (первой линии) XT.

При прогрессировании на фоне XТ первой линии (платинорефрактерный рак) возможна попытка монохимиотерапии одним из неплатиновых препаратов (табл. 4). Во всех других случаях, в том числе при неполном эффекте и наличии остаточной опухоли, целесообразно завершить лечение после 6 (максимум - 8) циклов ХТ и перейти к динамическому наблюдению или поддерживающей терапии до появления признаков прогрессирования заболевания. 
Таблица 3. Рекомендуемые режимы химиотерапии первичного рака яичников

\begin{tabular}{|c|c|}
\hline Показания & Режим химиотерапии \\
\hline \multirow{8}{*}{$\begin{array}{l}\text { 1) IA и IB стадии } \\
\text { при наличии факторов } \\
\text { высокого риска } \\
\text { рецидива болезни } \\
\text { (высокая степень } \\
\text { злокачественности, } \\
\text { светлоклеточный } \\
\text { гистологический тип, } \\
\text { отсутствие полного } \\
\text { хирургического } \\
\text { стадирования) } \\
\text { 2) IC-IV стадии }\end{array}$} & $\begin{array}{l}\text { Паклитаксел } 175 \text { мг/м² в/в в 1-й день, карбоплатин AUC } 6 \text { в/в в 1-й день } \\
\text { каждые } 3 \text { нед. }{ }^{1}\end{array}$ \\
\hline & $\begin{array}{l}\text { Паклитаксел } 175 \text { мг/м² в/в в 1-й день, цисплатин } 75 \text { мг/м² в/в в 1-й день } \\
\text { каждые } 3 \text { нед. }{ }^{1}\end{array}$ \\
\hline & $\begin{array}{l}\text { Паклитаксел } 80 \text { мг/м² в/в в 1-й, 8-й, 15-й дни, карбоплатин AUC } 6 \text { в/в в 1-й день } \\
\text { каждые } 3 \text { нед. }{ }^{1}\end{array}$ \\
\hline & $\begin{array}{l}\text { Доцетаксел } 75 \text { мг/м² в/в в 1-й день, цисплатин } 75 \text { мг/м² в/в в 1-й день } \\
\text { каждые } 3 \text { нед. }{ }^{1}\end{array}$ \\
\hline & Паклитаксел 60 мг/м² в/в еженедельно, карбоплатин AUC 2 в/в еженедельно \\
\hline & $\begin{array}{l}\text { Паклитаксел } 135 \text { мг/м² в/в в 1-й день, цисплатин } 75 \text { мг/м² внутрибрюшинно } \\
\text { во 2-й день, паклитаксел } 60 \text { мг/м² внутрибрюшинно в 8-й день каждые } 3 \text { нед. } \\
\text { (допустима замена цисплатина на карбоплатин AUC6 внутрибрюшинно во 2-й день)² }\end{array}$ \\
\hline & $\begin{array}{l}\text { Доксорубицин } 50 \text { мг/м² в/в в 1-й день, карбоплатин AUC } 6 \text { в/в в 1-й день } \\
\text { 21-дневного курса }\end{array}$ \\
\hline & Доксорубицин 50 мг/м² в/в, цисплатин 75 мг/м² в/в в 1-й день 21-дневного курса \\
\hline
\end{tabular}

1 у больных с III-IV стадиями и отсутствием мутаций BRCA1/2 к XT может быть добавлен бевацизумаб в следующих случаях: 1) при наличии резидуальных опухолевых узлов >10 мм; 2) при невозможности выполнения циторедуктивной операции (в данный момент и в перспективе); 3) при IV стадии. Не следует использовать бевацизумаб при проведении предоперационной ХТ. Введение бевацизумаба (7,5 или 15 мг/кг в/в 1 раз в 3 нед.) может быть начато с первого или второго курса ХТ и продолжено после ее завершения до общего количества 18-22 введения (или до прогрессирования, если оно наступит раньще).

2 Внутрибрюшинная XT используется при II-IV стадии после выполнения первичной циторедуктивной операции в оптимальном объеме (m. е. с максимальным размером остаточных опухолевых узлов $\leq 10$ мм) и требует предварительной имплантации внутрибрюшинного порта.

${ }^{3}$ Комбинация СР (производное платины и циклофосфамид) не рекомендуется в связи с низкой клинической эффективностью.

\subsection{2. ПодАерживающая терапия после химиотерапии первой линии}

\subsubsection{1. Бевацизумаб}

При назначении бевацизумаба в сочетании с ХТ возможно его продолжение в качестве поддерживающего лечения в дозах 7,5 или 15 мг/кг в/в 1 раз в 3 нед. до общего количества 1822 в ведения (или до прогрессирования, если оно наступит раньше). Если в процессе XТ первой линии в сочетании с бевацизумабом у больной была выявлена мутация генов BRCA1/2, рекомендовано перевести больную на поддерживающее лечение олапарибом, отменив бевацизумаб'.

1 Режим/nрепарат зарегистрирован в РФ, но не входит в клинические рекомендации, одобренные Минздравом РФ. 


\subsubsection{2. Олапариб}

Пациенткам с серозным РЯ высокой степени злокачественности III-IV стадий рекомендовано назначение поддерживающей терапии олапарибом при соблюдении следующих условий:

- наличие герминальной или соматической мутаций в генах BRCA1/2;

- противоопухолевый ответ (полный или частичный) на XТ первой линии.

Поддерживающая терапия олапарибом (в таблетках) проводится в дозе 300 мг ×2 раза в сутки внутрь в течение 2-х лет или до прогрессирования или непереносимой токсичности. При сохранении частичного ответа через 2 года после начала терапии лечение может быть продолжено на период свыше 2 лет, если, по мнению врача, это может принести пользу пациентке. Желательно начать поддерживающую терапию олапарибом в течение 8 нед. после окончания XT.

\subsubsection{3. Гормонотерапия}

В случае серозного рака низкой степени злокачественности II-IV стадий после окончания XТ возможна поддерживающая эндокринная терапия ингибиторами ароматазы (в стандартных дозах) или тамоксифеном 20-40 мг/сут. в течение 3 лет или до прогрессирования. Необходимость определения экспрессии РЭ и РП в опухоли для оценки предполагаемой чувствительности к эндокринной терапии не доказана и не является обязательной.

\section{3. Наблюдение после первичного лечения}

Принципы наблюдения за больными РЯ после первичного лечения до конца не определены. Результаты крупного международного исследования MRC OV-05 продемонстрировали отсутствие необходимости тщательного инструментального обследования сцелью максимально раннего выявления рецидива. ХТ второй линии целесообразно начинать при появлении клинических симптомов. Раннее начало ХТ второй линии, например, при маркерном рецидиве, не увеличивает продолжительность жизни, но ухудшает ее качество за счет побочных эффектов лечения.

По этой причине члены рабочей группы предлагают следующий алгоритм наблюдения за больными:

- определение концентрации СА125 в плазме крови, осмотр гинеколога и УзИ брюшной полости и малого таза, забрюшинного пространства 1 раз в 3 мес. в течение первых 2 лет, 1 раз в 4 мес.- в течение 3-го года, далее - каждые 6 мес.;

- углубленное обследование (КТ брюшной полости и малого таза, рентгенография/КТ грудной клетки, ПЭТ/КТ в случае планирования хирургического лечения) показано при росте СА125, появлении жалоб или выявлении патологии при гинекологическом осмотре или УЗИ. 


\section{4. ЛЕЧЕНИЕ РЕЦИДИВОВ РАКА ЯИЧНИКОВ, РАКА БРЮШИНЫ И РАКА МАТОЧНЫХ ТРУБ}

\section{1. Критерии прогрессирования и показания к началу лекарственной терапии}

Прогрессированием РЯ является наличие любого из перечисленных ниже критериев:

- клинические (осмотр, пальпация) или радиологические (данные инструментального обследования) признаки прогрессирования;

- повышение уровня СА125, подтвержденное двумя последовательными анализами, выполненными с интервалом не менее 1 недели, с соблюдением следующих условий:

- повышение концентрации СА125 в 2 раза выше ВГН при нормальных предшествующих значениях;

- повышение уровня СА125 в 2 раза выше своего наименьшего значения, зарегистрированного во время лечения и наблюдения (при отсутствии нормализации маркера).

Признаки прогрессирования НЕ ЯВЛЯЮТСЯ АБСОЛЮТНЫМ ПОКАЗАНИЕМ для начала новой линии ХT, а служат для оценки эффективности предыдущего лечения, В т. ч. определения длительности бесплатинового интервала. Показанием для ХТ второй и последующих линий является рост опухолевых очагов и наличие жалоб, связанных с опухолевым ростом.

\section{2. Повторные циторедуктивные вмешательства}

У некоторых больных с рецидивами РЯ могут выполняться повторные циторедуктивные вмешательства. После ранее выполненной полной первичной циторедукции может обсуждаться хирургическое лечение рецидивов РЯ при соблюдении следующих условий:

- длительность бесплатинового интервала >6 мес.;

- наличие одной или нескольких рецидивных опухолей;

- отсутствие клинически значимого асцита;

- удовлетворительное общее состояние больной (по шкале ECOG 0 баллов).

Повторная циторедуктивная операция целесообразна только в том случае, если хирург считает возможным выполнение полной циторедукции (без макроскопически определяемой остаточной опухоли). Хирургическое вмешательство следует планировать до начала ХТ по поводу рецидива. Сроки выполнения хирургических вмешательств по поводу рецидива рака low-grade определяются индивидуально'.

1 Режим/nрепарат зарегистрирован в РФ, но не входит в клинические рекомендации, одобренные Минздравом РФ. 


\section{3. Выбор режима химиотерапии}

Выбор режима ХТ основан на оценке предполагаемой чувствительности к производным платины, определяемой косвенно по длительности бесплатинового интервала, который рассчитывается от даты последнего введения производного платины до даты прогрессирования. Выделяют следующие типы рецидивов:

- платиночувствительный: длительность бесплатинового интервала > 6 мес.;

- платинорезистентный: длительность бесплатинового интервала < 6 мес.;

- платинорефрактерный: прогрессирование во время платиносодержащей ХТ первой линии либо в течение 1 мес. после ее завершения.

При платиночувствительном рецидиве показано назначение комбинации цисплатина или карбоплатина с другим противоопухолевым препаратом, не использовавшимся ранее (табл. 4). При длительности бесплатинового интервала >24 мес. возможно повторное назначение комбинации производного платины и таксанов.

При длительности бесплатинового интервала менее 6 мес. и при невыраженных симптомах онкологического процесса рекомендуется бесплатиновая монохимиотерапия. При прогрессировании опухоли в дальнейшем повторное применение платиносодержащей ХТ является предпочтительным, если ранее не было зарегистрировано прогрессирования опухоли во время ХТ производными платины. При выраженных симптомах, связанных с прогрессированием опухолевого процесса, или при наличии мутации в генах BRCA целесообразно сразу возобновить платиносодержащую ХТ, если ранее не было зарегистрировано прогрессирования опухоли во время ХТ производными платины (табл. 4).

В случае прогрессирования заболевания в процессе двух последовательных линий XT целесообразность продолжения лечения сомнительна. В этом случае рекомендуется рассмотреть возможность проведения симптоматической терапии или включения пациентки в клинические исследования.

При использовании в первой линии ХТ бестаксановой комбинации включение таксанов в режим второй линии является предпочтительным (в комбинации с производными платины - при платиночувствительном рецидиве и монохимиотерапия таксанами - при платинорезистентном рецидиве). Рекомендуемое количество циклов ХТ второй линии составляет 4-6 при наличии эффекта и удовлетворительной переносимости. Вопрос о продлении XТ после 6 циклов следует рассматривать индивидуально. Метрономная терапия проводится до прогрессирования или непереносимой токсичности.

У больных с аденокарциномой low-grade возможно назначение ГТ в качестве самостоятельной линии лечения после повторной циторедуктивной операции по поводу рецидива или при прогрессировании заболевания. Лечение проводится до прогрессирования или непереносимой токсичности'.

Алгоритм терапии последующих рецидивов аналогичен описанному выше.

1 Режим/nрепарат зарегистрирован в РФ, но не входит в клинические рекомендации, одобренные Минздравом РФ. 
Таблица 4. Рекомендуемые режимы химиотерапии рецидивов рака яичников

\begin{tabular}{|c|c|}
\hline & Режим химиотерапии \\
\hline $\begin{array}{l}\text { Комбинирован- } \\
\text { ная химио- } \\
\text { терапия }\end{array}$ & 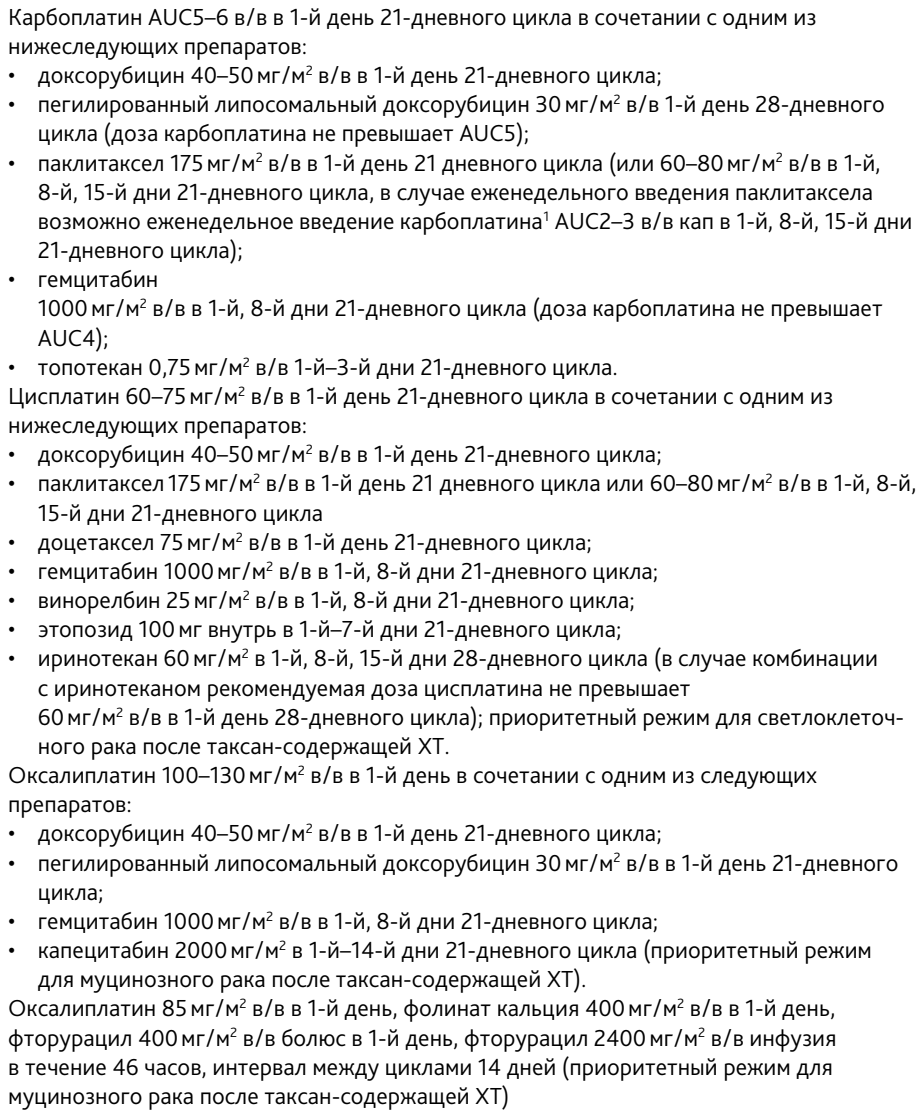 \\
\hline
\end{tabular}




\begin{tabular}{|c|c|}
\hline & Режим химиотерапии \\
\hline $\begin{array}{l}\text { Монохимио- } \\
\text { терапия, } \\
\text { метрономная } \\
\text { химиотерапия, } \\
\text { иммунотерапия }\end{array}$ & 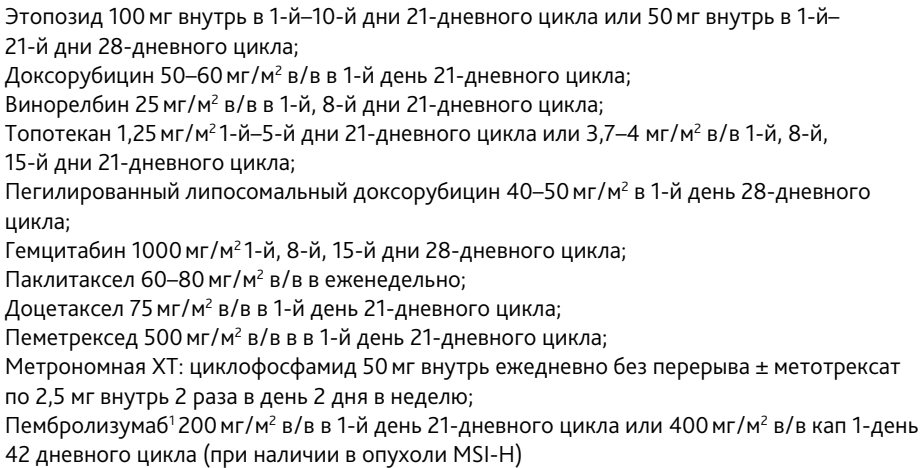 \\
\hline $\begin{array}{l}\text { Эндокрино- } \\
\text { терапия }\end{array}$ & $\begin{array}{l}\text { Летрозол 2,5 мг в сутки внутрь ежедневно; } \\
\text { Анастрозол } 1 \text { мг в сутки внутрь ежедневно; } \\
\text { Тамоксифен 20-40 мг в сутки внутрь ежедневно; } \\
\text { Мегестрол } 160 \text { мг в сутки внутрь ежедневно }\end{array}$ \\
\hline
\end{tabular}

1 Режим/nрепарат зарегистрирован в РФ, но не входит в клинические рекомендации, одобренные МинздравомРФ.

\section{4. Поддерживающая терапия при рецидивах рака яичников}

\subsection{1. Бевацизумаб}

Добавление бевацизумаба (7,5 или 15 мг/кг в/в 1 раз в 3 нед.; 5 или 10 мг/кг каждые 2 недели в зависимости от режима ХTํ) показано больным с рецидивами заболевания. Назначение поддерживающей терапии бевацизумабом у больных с мутацией BRCA1/2 рекомендовано после получения PARP-ингибитора в предыдущих линиях в поддерживающем режиме. Терапию бевацизумабом следует начинать с 1-2 цикла ХT. После окончания ХT введение бевацизумаба следует продолжить до прогрессирования заболевания или неприемлемой токсичности.

\subsubsection{PARP-ингибитор олапариб}

Назначение PARP-ингибитора олапариба в качестве поддерживающей терапии рекомендуется при наличии всех следующих критериев:

- карцинома высокой степени злокачественности при патогенной мутации (герминальной или соматической) в генах BRCA1/2;

- объективный эффект после проведения последней платиносодержащей ХT, назначенной по поводу платиночувствительного рецидива.

При лечении рецидивов РЯ олапариб может быть назначен вне зависимости от мутационного статуса генов BRCA1/2. В то же время, члены рабочей группы отмечают, что 
в настоящее время отсутствует убедительная доказательная база, поддерживающая применение олапариба у всех пациенток с «диким» типом указанных генов. При лечении данной категории больных возможность назначения олапариба следует рассматривать в тех случаях, когда течение заболевания свидетельствует о повышенной чувствительности опухолевых клеток к ДНК-повреждающим агентам (например, у больных серозной и эндометриоидной аденокарциномой high grade с длительным бесплатиновым интервалом и/или высокой чувствительностьюк препаратам платины).

Прием олапариба (по 400 мг 2 раза в сутки внутрь в лекарственной форме «капсулы для приема внутрь» или 300 мг 2 раза в сутки внутрь в лекарственной форме «таблетки для приема внутрь») желательно начать в течение 8 нед. после окончания платиносодержащей $\mathrm{XT}$, лечение препаратом следует продолжать до прогрессирования или возникновения непереносимой токсичности.

\section{5. Лучевая терапия}

Некоторые несерозные гистологические типы, например, светлоклеточный, муцинозный или эндометриоидный, во многих случаях локализованы в тазу, а метастазирование может быть ограничено регионарными лимфатическими узлами. При развитии резистентности несерозных гистотипов к лекарственной терапии возможно применение ЛТ рецидивных очагов и метастазов.

\section{6. Профилактика реакций гиперчувствительности}

При лечении пациенток с анамнезом аллергических реакций (реакций гиперчувствительности) на платиновые агенты рекомендуется оценить возможность продолжения терапии платиновыми препаратами, используя следующий алгоритм.

1) Оценить выраженность аллергической реакции. В случае развития аллергической реакции степени 1-3 по шкале СТС-АЕ следует рассмотреть возможность продолжения терапии с использованием десенсибилизирующей схемы введения препарата. В случае развития аллергической реакции 4 степени по СТСАЕ (жизнеугрожающая реакция гиперчувствительности) препарат, вызвавший её развитие, должен быть отменен и не назначаться в последующем до консультации специалиста, имеющего опыт в проведении десенсибилизирующей терапии (аллерголог или онколог-химиотерапевт).

2) При выборе протокола десенсибилизации следует руководствоваться данными специальной литературы. Протокол десенсибилизации должен включать дробное введение препарата, вызвавшего аллергическую реакцию, и профилактическое применение препаратов, снижающих риск гиперчувствительности (Н1-гистаминоблокаторы, ГКС). При лечении пациенток с реакциями гиперчувствительности обязательно следование выбранному протоколу десенсибилизации при проведении каждого последующего курса лечения.

3) Перед проведением терапии с использованием десенсибилизирующей схемы следует убедиться, что персонал клиники знаком с симптомами аллергических реакций, 
порядком действий в случае их возникновения. Должен быть обеспечен быстрый доступ к необходимым препаратам (Н1-гистаминоблокаторы, ГКС, адреналин) и/или к реанимационной службе.

4) При повторном развитии реакций гиперчувствительности, несмотря на следование протоколу десенсибилизации, дальнейшие решения по тактике лечения следует принимать индивидуально, с учетом соотношения риска и пользы от продолжения терапии, а также выраженности реакции гиперчувствительности.

5) В некоторых случаях может быть целесообразной замена платинового агента, вызвавшего реакцию гиперчувствительности, на другой препарат того же класса (например, карбоплатина на цисплатин или оксалиплатин). При замене препарата следует учитывать спектр его токсичности и эффективности в составе используемого режима лечения.

В настоящее время отсутствуют данные, демонстрирующие преимущество какого-либо конкретного протокола перед другими возможными схемами десенсибилизации. В качестве примера можно привести следующий алгоритм действий (протокол десенсибилизации к карбоплатину):

а) фексофенадин 60 мг (или аналогичный Н1-блокатор) внутрь 2 раза в сутки, начиная за 24 ч до проведения X T; 120 мг внутрь за 3 часа до XT;

6) дексаметазон 20 мг в/м, в/в или внутрь за 12 ч до XT; 20 мг - за 3 часа до XT; 20 мг в/в - за 30 мин. до ХТ;

в) фамотидин 20 мг (или аналогичный Н2-блокатор) в/в или внутрь за 30 мин. до ХT;

г) дифенгидрамин 50 мг (или аналогичный Н1-блокатор) в/в за 30 мин. До ХТ, далее 25 мг каждые 4 часа - 3 введения;

д) дробное введение карбоплатина (суммарная доза препарата определяется по формуле Кальверта), доза препарата разводится дробно на 4 флакона 0,9\% раствора $\mathrm{NaCl}$ :

- 1 флакон - 1/1000 суммарной дозы на 30 мл 0,9\% $\mathrm{NaCl}$ в/в капельно в течение 30 мин.;

- 2 флакон - 1/100 суммарной дозы на 50 мл 0,9\% $\mathrm{NaCl}$ в/в капельно в течение 30 мин.;

- 3 флакон - 1/10 суммарной дозы на 100 мл 0,9\% $\mathrm{NaCl}$ в/в капельно в течение 60 мин.;

- 4 флакон - вся оставшаяся доза препарата на 250 мл 0,9\% NaCl в/в капельно в течение $\geq 90$ мин. 